\title{
The Role of Clinical Examination in Predicting Relevant MRI Findings in Acute Knee Injuries: A Retrospective Study
}

\author{
Raghuram Thonse, MBBS, MS, DNB, FRCSEd, FRCS 3 \\ ${ }^{1}$ Department of Trauma and Orthopaedics, Stepping Hill Hospital, \\ Stockport, United Kingdom \\ ${ }^{2}$ Department of Trauma and Orthopaedics, Manchester Royal \\ Infirmary, Manchester, United Kingdom \\ ${ }^{3}$ Department of Trauma and Orthopaedics, Countess of Chester \\ Hospital NHS Foundation Trust, Chester, Cheshire West and Chester, \\ United Kingdom
}

Daoud Makki, MD, MRCS, FRCS ${ }^{1}$ Saleem Mastan, MBChB, MRCS ${ }^{2}$

J Knee Surg 2019;32:280-283.

\author{
Daniel Ness, MBChB, MRCS 3
}

\begin{abstract}
Address for correspondence Saleem Mastan, MBChB, MRCS, Department of Trauma and Orthopaedics, Manchester Royal Infirmary, Oxford Road, Manchester M13 9WL, United Kingdom (e-mail: saleemmastan941@gmail.com).
\end{abstract}

\begin{abstract}
Keywords

- acute knee injury

- MRI

- clinical examination

The objective of this study was to delineate the usefulness of clinical examination and magnetic resonance imaging (MRI) in acute knee injuries. We aim to establish whether the time period post acute knee injury is related to the diagnostic accuracy of clinical examination and to investigate the strength of specific clinical examination findings in predicting a clinically relevant MRI abnormality. Seventy patients were referred to fracture clinic with an acute knee injury who subsequently went on to be investigated with MRI over 12 months. These patients were retrospectively analyzed looking at the time period they were reviewed, the components that were assessed at physical examination, and the results of their eventual MRI scan looking for any correlation. A greater proportion of patients who were examined at 2 weeks had relevant positive findings on MRI scan, $p=0.03$. Range of movement and lateral joint line tenderness were not associated with a positive MRI scan at any period after injury. The presence of a moderate to large effusion was not associated with an MRI abnormality if the examination was within 2 weeks of injury but was if present 2 weeks after injury, $p=0.0001$. Range of movement should not form part of the decision making on whether an injury should be investigated with MRI. Joint effusion in isolation within 2 weeks after injury should not be an indication for MRI but a repeat clinical examination in 2 weeks, where if still present, should be investigated with MRI.
\end{abstract}

Magnetic resonance imaging (MRI) provides a useful noninvasive investigation to diagnose soft-tissue injuries, without the need for any operative intervention. It is, however, a costly intervention so resources need to be appropriately allocated. The routine use for knee injuries should be avoided. Some studies have shown that a thorough clinical examination can be as good if not better than MRI in diagnosing certain injuries of the knee. ${ }^{1}$ Felli et al noted

received

September 12, 2017

accepted after revision

February 25, 2018

published online

April 13, 2018 the clinical accuracy of medial meniscus rupture was $84 \%$, using arthroscopy as the gold standard. ${ }^{2}$ There are also studies that look at the sensitivity of certain knee tests for certain knee injury. ${ }^{3}$ However, there is limited evidence that compares clinical knee examination to the results of an MRI scan. Furthermore, no study exists that looks at the usefulness of clinical examination in predicting a relevant MRI abnormality is related to the time after the injury they are
Copyright $\odot 2019$ by Thieme Medical Publishers, Inc., 333 Seventh Avenue, New York, NY 10001, USA. Tel: +1(212) 584-4662.
DOI https://doi.org/ 10.1055/s-0038-1641154. ISSN 1538-8506. 
examined. A more appropriate temporal clinical knee examination will lead to the more efficient use of MRI.

\section{Methods}

All patients referred to fracture clinic from accident and emergency department between January and December 2013 with acute soft-tissue knee injuries and who subsequently underwent MRI scans, were retrospectively analyzed. Patients with preexisting or recurrent knee problems were excluded from the study. Data collection included the mechanism of injury, time from injury to first assessment, clinical examination, and the suspected clinical diagnosis. We compared the clinical findings to those that were revealed on the MRI scan later. We investigated whether the findings on the MRI scan and their relevance correlated to the timing of clinical examination (within 2 weeks or after 2 weeks from the injury). Documentation of clinical findings was uniform following a template that assesses a set of variables when pain permits. The significance of each clinical variable was correlated with the abnormalities detected in the scan and the positive and negative predictive values of certain variable were calculated.

\section{Results}

In total, 70 patients who fitted the criteria of first presentation with acute knee injury were studied. The mean age of patients was 28 years (range, 13-52 years). There were 44 males (62.9\%) and 26 females (37.1\%). The mechanism of injury was sports-related in 25 (35.7\%) patients and $2(2.9 \%)$ were involved in road-traffic accidents. The remaining patients were all minor trauma such as twisting injury while walking, running, and going on stairs. Forty-seven (67.1\%) of the patients were clinically reviewed in under 2 weeks after their injury and 23 (32.9\%) were reviewed over 2 weeks after their injury.

The decision was taken to investigate with MRI after clinical examination in 58 (82.9\%) of the patients, whereas $12(17.1 \%)$ of the patients were brought back for a repeat clinical examination before a MRI was ordered. As the level of pain permitted, clinical examination included the assessment of the following variables: medial joint line tenderness in all patients (100\%), lateral joint line tenderness in 61 patients (87.1\%), range of movement in 68 patients (97.1\%), effusion in 65 patients (92.9\%), Lachman test in 50 patients (71.4\%), posterior draw test in 38 patients (54\%), and laxity in 32 patients (46\%) (-Fig. 1).

\section{Time from Injury to Clinical Assessment}

Of the 47 patients who were reviewed clinically in under 2 weeks after injury, 34 (72.3\%) had a positive MRI finding of any description. Among the 23 patients who were assessed over 2 weeks after their injury, 20 (86.9\%) patients had a positive MRI finding. There was no statistical significance between the two groups looking at all abnormalities on MRI, $p=0.2($ - Fig. 2).

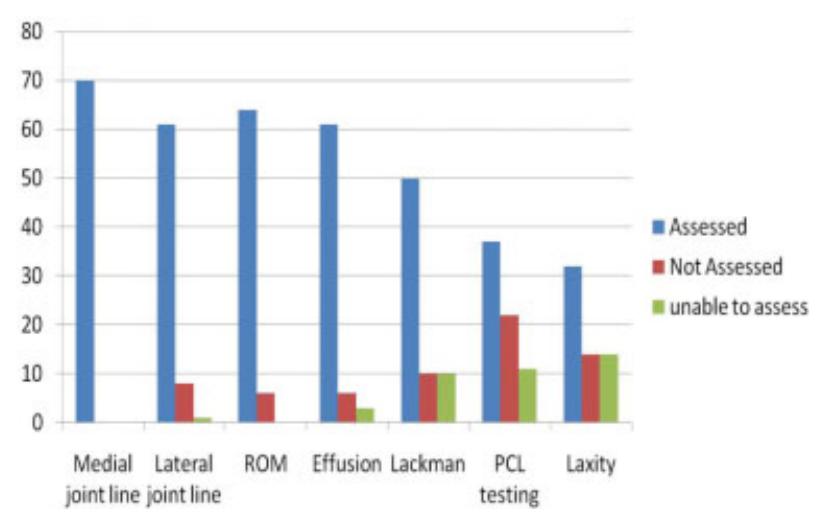

Fig. 1 Clinical examination assessment of all patients in study.

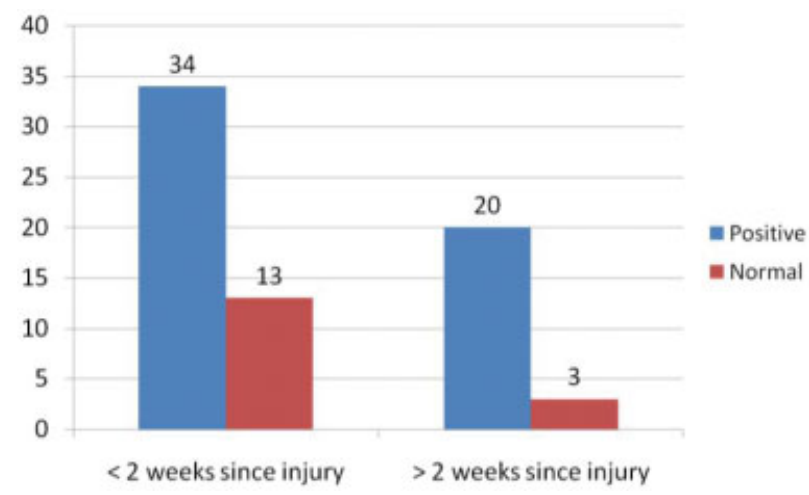

Fig. 2 Positive magnetic resonance imaging (MRI) findings, whether relevant or not, in the two groups identified.

However, when looking at relevant MRI findings, there was a statistically significant difference between the two groups. Relevant MRI findings were defined as related to their clinical presentation. Of the patients who were assessed in under 2 weeks, $14(29.8 \%)$ had a positive relevant MRI finding. Of the patients who were assessed 2 weeks or more after their injury, 13 (56.5\%) had a positive relevant MRI finding. There was a statistically significant difference in examination 2 weeks after injury compared with examination within 2 weeks in predicting a MRI abnormality (-Fig. 3).

\section{Clinical Variables}

\section{1-Range of Movement}

In $68(97.1 \%)$ patients, range of movement at the knee joint was assessed. Forty-four (64.7\%) of the patients had a reduced range of movement at the time of assessment and 24 (35.3\%) of the patients had a normal range of movement. There was no statistically significant difference between the two groups of patients in relation to a positive soft-tissue injury of the knee on MRI, $p=0.99$ ( - Fig. 4.) This was the case irrespective of time after injury. 
282 The Role of Clinical Examination in Predicting Relevant MRI Findings in Acute Knee Injuries Makki et al.

\begin{tabular}{|c|c|c|c|}
\hline & $\begin{array}{l}\text { Assessed within } 2 \\
\text { weeks ( } 47 \\
\text { patients) }\end{array}$ & $\begin{array}{l}\text { Assessed after } 2 \text { weeks ( } 23 \\
\text { patients) }\end{array}$ & $P$-Value \\
\hline Positive relevant & 14 & 13 & $0.03^{a}$ \\
\hline MRI results & & & \\
\hline
\end{tabular}

Fig. 3 Difference between relevant magnetic resonance imaging findings in the two groups. ${ }^{a}$ Fisher's exact test.

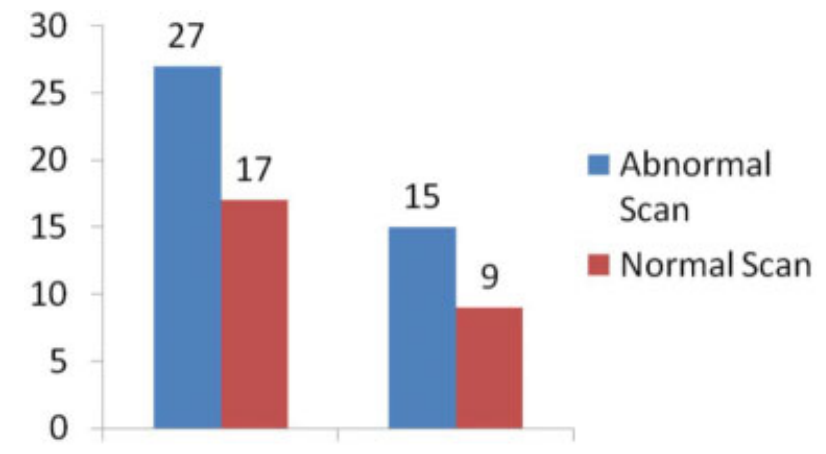

Reduced ROM Normal ROM

Fig. 4 Range of movement correlated to a positive magnetic resonance imaging scan.

\section{Lateral Joint Line Tenderness}

In $61(87.1 \%)$ of the patients, lateral joint line tenderness was assessed. Nineteen (27.1\%) of these patients had lateral joint line tenderness and 42 (68.9\%) did not (-Fig. 5). Although a greater proportion of the group with lateral tenderness had a positive MRI scan (31.5\% compared with 16.7\%), there was no statistical significance between the groups; $p=0.1649$. This was the case irrespective of time assessed after injury.

\section{Lachman Test}

In $50(71.4 \%)$ of the patients, Lachman test to assess the anterior cruciate ligament (ACL) was performed. Eighteen patients had a positive Lachman test with 11 (61.1\%) having a ruptured ACL on MRI. Thirty-two patients tested negative for

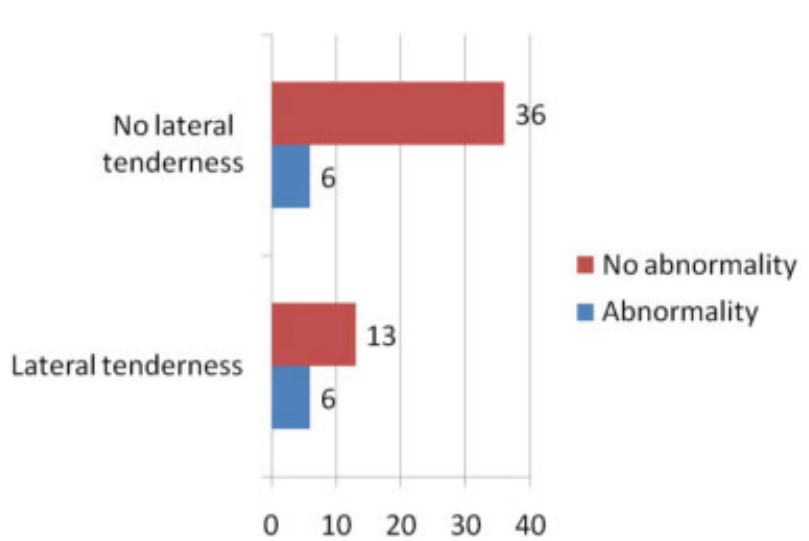

Fig. 5 Lateral joint line tenderness correlated to a positive magnetic resonance imaging scan.
Lachman's and 4 of these patients (12.5\%) had a ruptured ACL on MRI (-Fig. 6). There was a statistical significance between these groups; $p=0.0008$. This significance was present at any time after injury. The test was shown to have a high specificity (80\%) and a high negative predictive value (87.5\%).

\section{Effusions}

The presence or absence of an effusion was assessed in 65 (92.9\%) of the patients. Thirty (46.1\%) of the patients had a moderate to large effusion at the time they were examined (-Fig. 7). Overall, the presence of a moderate to large effusion did correlate to a positive MRI finding; $p=0.01$. There was no

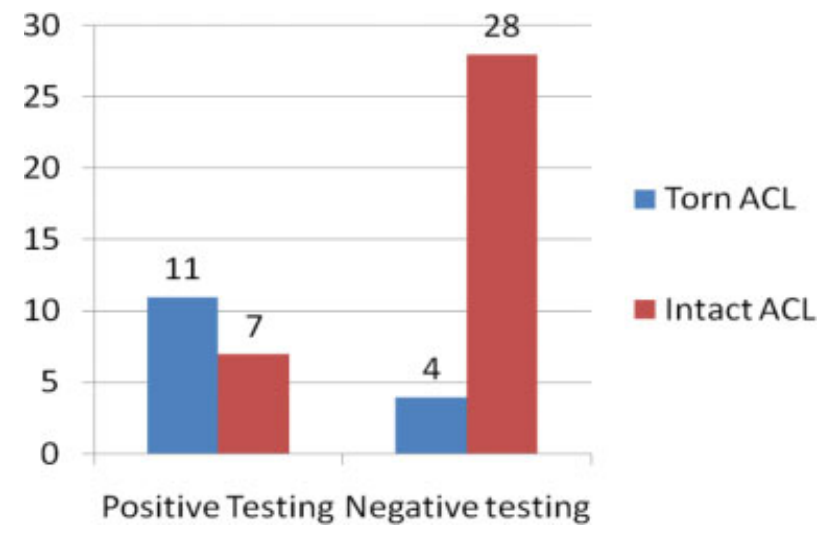

Fig. 6 Lachman's test correlated to positive magnetic resonance imaging scan.

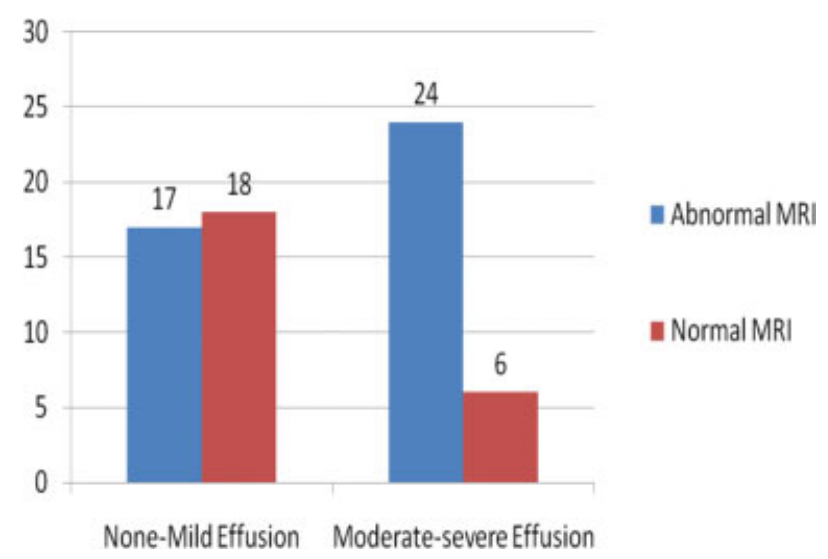

Fig. 7 Effusion correlating to positive magnetic resonance imaging scan. 


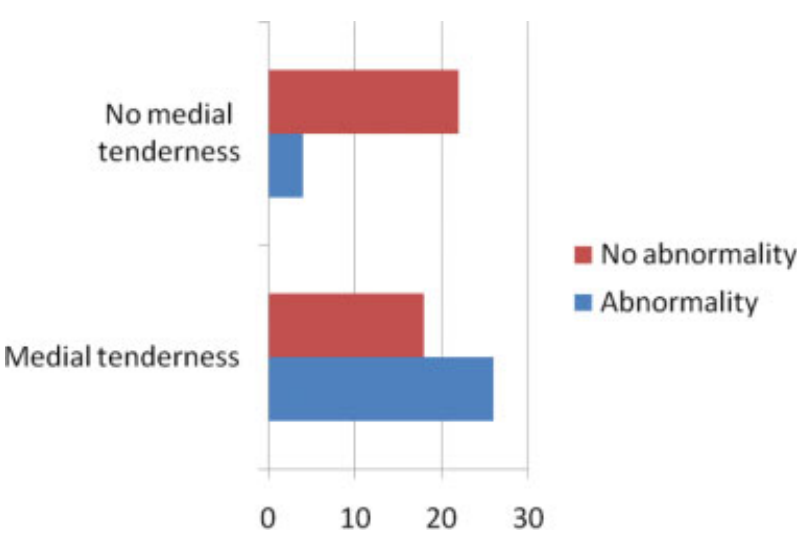

Fig. 8 Medial joint line tenderness correlating to magnetic resonance imaging (MRI) abnormalities.

statistical significance at a presence of an effusion if the knee was examined at less than 2 weeks, but it was significant if the effusion was present at an examination more than 2 weeks after the injury; $p$-value $=0.0001$.

\section{Medial Joint Line Tenderness}

Seventy (100\%) patients were assessed for medial joint line tenderness (-Fig. 8). Forty-four (62.9\%) of the patients had medial joint line tenderness, which was associated with MRI abnormalities irrespective of how long they were examined after injury; $p=0.0004$. Medial tenderness was more sensitive than specific, with a negative predictive value of $76 \%$ and a positive predictive value of $61 \%$ (-Table 1 ).

\section{Discussion}

The findings correlate to previous studies on the usefulness of each component of the knee examination. ${ }^{3}$ Lachman test has shown again to be highly specific and sensitive for ACL injuries, with joint line tenderness being sensitive but not specific for meniscal injuries. The relevance of timing in the development of an effusion is established, with rapid onset highly likely for a ligamentous injury. ${ }^{4}$ This study is unique in demonstrating that continued presence of an effusion 2 weeks after the onset is also associated with a structural injury that can be diagnosed on a MRI scan. On the basis of our findings, we therefore recommend a repeat clinical review 2 weeks after the initial injury if effusion is the only positive feature on examination. Should it still be present, it should be investigated by MRI.

Range of movement was not associated with a MRI abnormality at any stage after a knee injury. This would
Table 1 Overall statistical significance of the components of the knee examination

\begin{tabular}{|l|l|l|l|}
\hline & $\begin{array}{l}\text { Patients } \\
\text { assessed within } \\
\text { 2 weeks since } \\
\text { injury }\end{array}$ & $\begin{array}{l}\text { Patients } \\
\text { assessed } \\
\text { 2 weeks } \\
\text { since injury }\end{array}$ & Overall \\
\hline ROM & - & - & - \\
\hline $\begin{array}{l}\text { Lateral } \\
\text { tenderness }\end{array}$ & - & - & - \\
\hline Effusions & - & + & + \\
\hline ACL testing & + & + & + \\
\hline $\begin{array}{l}\text { Medial } \\
\text { tenderness }\end{array}$ & + & + & + \\
\hline
\end{tabular}

Abbreviations: ACL, anterior cruciate ligament; ROM, range of motion.

suggest that range of movement is not useful in diagnosing or excluding soft-tissue knee injuries and should not in isolation form part of the clinician's decision making to investigate the injury further.

Our study does have limitations. We have assumed MRI to be a gold standard for diagnosis, which is widely documented not to be the case. ${ }^{5,6}$ We would recommend further high-level studies looking at the accuracy of clinical investigations compared with arthroscopic gold standard investigations.

Conflict of Interest

None.

\section{References}

1 Rayan F, Bhonsle S, Shukla DD. Clinical, MRI, and arthroscopic correlation in meniscal and anterior cruciate ligament injuries. Int Orthop 2009;33(01):129-132

2 Felli L, Garlaschi G, Muda A, et al. Comparison of clinical, MRI and arthroscopic assessments of chronic ACL injuries, meniscal tears and cartilage defects. Musculoskelet Surg 2016;100(03):231-238

3 Malanga GA, Andrus S, Nadler SF, McLean J. Physical examination of the knee: a review of the original test description and scientific validity of common orthopedic tests. Arch Phys Med Rehabil 2003;84(04):592-603

4 Sternbach GL. Evaluation of the knee. J Emerg Med 1986;4(02): 133-143

5 Dufka FL, Lansdown DA, Zhang AL, Allen CR, Ma CB, Feeley BT. Accuracy of MRI evaluation of meniscus tears in the setting of ACL injuries. Knee 2016;23(03):460-464

6 Nam TS, Kim MK, Ahn JH. Accuracy of MRI evaluation of 206 meniscal tears in acute anterior cruciate ligament injuries. Arthroscopy 2014;30:475-482 